\title{
Editorial
}

\section{Embedded Systems for Mobile Sensors}

\author{
Marco Anisetti, ${ }^{1}$ Valerio Bellandi, ${ }^{1}$ Abdellah Chehri, ${ }^{2}$ Yurong Qian, ${ }^{3}$ and Gwanggil Jeon \\ ${ }^{1}$ Dipartimento di Informatica, Università degli Studi di Milano, Via Bramante 65, 26013 Crema, Italy \\ ${ }^{2}$ School of Electrical Engineering and Computer Science, University of Ottawa, 800 King Edward Avenue, \\ Ottawa, ON, Canada K1N 6N5 \\ ${ }^{3}$ Software College, Xinjiang University, 14 Sheng Li Road, Urumqi, Xinjiang 830008, China \\ ${ }^{4}$ Department of Embedded Systems Engineering, Incheon National University, 119 Academy-ro, Yeonsu-gu, \\ Incheon 406-772, Republic of Korea
}

Correspondence should be addressed to Gwanggil Jeon; gjeon@inu.ac.kr

Received 21 July 2016; Accepted 22 July 2016

Copyright (C) 2016 Marco Anisetti et al. This is an open access article distributed under the Creative Commons Attribution License, which permits unrestricted use, distribution, and reproduction in any medium, provided the original work is properly cited.

In the era of IoT, embedded systems based on mobile sensor ideas turn into increasingly compound and need understanding in various training tools such as signal processing, artificial intelligence, and multimedia communication. After a long period of computational capacity centralization, we are facing a period where the computation capacity is migrating back to the periphery of the systems and then to smart objects and sensors. Currently IoT is constituted of computationally heterogeneous devices for which the ability of executing preelaborations or complete processing is fundamental as well as the ability of doing them, keeping low power consumptions and maintaining a certain level of security. The combination of signal processing techniques for embedded systems is a principal and timely research in mobile sensors and communications. The articles contained in the present issue include both reviews and basic scientific studies focused on mobile multimedia communication using fundamental or applied signal processing methods for embedded systems and applications of IoT. This issue comprises the description of streams processing (e.g., images, video, and audio) for embedded systems involved in IoT as mobile sensors/aggregators including preprocessing for signal quality enhancement (e.g., images, noise reduction), security concerns (e.g., privacy), and evolution of communication protocols and mobile sensor network architectures.

Many challenges exist in constructing smart building/home system including interconnectivity issues between different technologies and the need of continuous connectivity between heterogeneous sensors. A smart home gateway taking responsibility for the connection between the network layer and the ubiquitous sensor network layer plays an important role in automated home Internet of Things system. The contribution of F. Ding et al. "A Smart Gateway Architecture for Improving Efficiency of Home Network Applications" proposes an integrated access gateway providing significant flexibility for user to configure and deploy supporting multiubiquitous sensor network. One of the basic issues for information collection and data processing in wireless sensor networks is the network coverage. The contribution of $\mathrm{F}$. Ding and A. Song "Development and Coverage Evaluation of ZigBee-Based Wireless Network Applications" presented coverage evaluation and efficient development of a ZigBeebased home network application based on adaptive weighted fusion (AWF) of all data of sensing nodes and a gatewaybased architecture to reexecute packet processing and then reported to the monitoring center.

Quality of Service (QoS) parameters such as bit rate and packet losses are good indicators for optimizing network services but not for characterizing user perception called Quality of Experience (QoE). The contribution of G. Sarwar et al. "QoS and QoE Aware N-Screen Multicast Service" proposed QoS and QoE-aware adaptive mapping of N-Screen devices to multicast groups at the application layer to ensure the visual quality requirements in varying network conditions. 
Power consumption is another crucial aspect in IoT and mobile sensor network systems. The contribution of A. Ahmad et al. "Context-Aware Mobile Sensors for Sensing Discrete Events in Smart Environment" proposed a smart home architecture introducing the notion of context-aware low power mobile sensors sharing the same communication model based on IoT paradigm. The author tested the performances on Hadoop and the energy consumption of the sensors showing the advantages of their solution.

The mobile phone is becoming a powerful environmental sensing unit that represents a strong support for several applications domains, ranging from traffic management to advertisement and social studies. However, the limited battery capacity of mobile devices represents a big obstacle. The contribution of M. Boukhechba et al. "Energy Optimization for Outdoor Activity Recognition" presented an approach for the recognition of users' outdoor activities able to keep mobile resources consumption under control behaving variably in function of users' behaviors and the remaining battery level.

Dedicated sensors can harvest the energy from the environment, but they cannot charge and discharge at the same time. The contribution of X.-F. Zhang and C.-C. Yin "Energy Harvesting and Information Transmission Protocol in Sensors Networks" studied a wireless system under energy harvesting conditions proposing a method to optimize the system outage performance via finding the optimal save ratio.

Preprocessing of sensors data is a fundamental step for improving the quality of the signal to be effectively used in the following steps of the target application (e.g., image recognition, speech recognition, and human computer interaction systems). More specifically preprocessing for noise reduction has a paramount importance. Noise in video streams impact negatively any type of processing activities aimed at detecting objects. Often object detection uses edge detection as one of the basic features for the recognition. Considering the high computational complexity of applying edge detection and filtering to $1080 \mathrm{p}$ video, hardware implementation on reconfigurable hardware fabric is necessary. The contribution of I. Yoon et al. “Zynq-Based Reconfigurable System for RealTime Edge Detection of Noisy Video Sequences" proposed an embedded system utilizes dynamic reconfiguration features of Zynq SoC to perform partial reconfiguration of different filter bitstreams during run-time according to the detected noise density level in the incoming video frames.

Noise estimation algorithms are essential components of many modern mobile communications. The contribution of S. Lee and G. Lee "Noise Estimation and Suppression Using Nonlinear Function with A Priori Speech Absence Probability in Speech Enhancement" proposed a noise-based compensation of minimum statistics method for speech enhancement in highly nonstationary noisy environments using a nonlinear function and a priori speech absence probability. Active noise cancellation is a technique based on adaptive feedforward control mostly to superpose an artificial sound to an unwanted disturbance noise. The contribution of Y.-S. Lee et al. "Length Variation Effect of the Impulse Response Model of a Secondary Path in Embedded Control" investigated on the length variation effect of the impulse response function for the secondary path model in active noise control using an embedded control board.

The contribution of S. Ryu and Y.-S. Lee "Characteristics of Relocated Quiet Zones Using Virtual Microphone Algorithm in an Active Headrest System" proposed a theoretical and experimental investigation on the characteristics of the relocated zone of quiet as effective antinoise solution using virtual microphone based FxLMS algorithm suitable to be embedded in a real-time digital controller for an active headrest system.

The principal and most challenging requirement for image enlargement methods in embedded systems is keeping a good balance between performance, low computational cost, and low memory usage. The contribution of $\mathrm{H}$. Hua et al. "An efficient Image Enlargement Method for Image Sensors of Mobile in Embedded Systems" proposes an efficient image enlargement method based on different kinds of features for different morphologies with different approaches and the relative learned dictionaries for efficient image representation. The authors adopt clustering approach and projection matrix on dictionaries for improving speed and memory usage.

Seaming finding is an important step for creating panorama images for smoothing the differences observed at boundaries between stitched images. The contribution of $\mathrm{J}$. Jeong and K. Jun "A Novel Seam Finding Method Using Downscaling and Cost for Image Stitching" proposed an improved seam finding method based on a cost function to determine how each pixel affects stitching and a downscaled version of overlapped area to approximate a seam and then interpolate the seam to the original region.

Privacy-preserving elaboration and transmission of sensing data have drawn much attention recently. Encryption of a digital image is fundamental in applications of body area networks where the captured image may include a number of privacy issues. Some of the disadvantages of the past encryption methods are the small key space and low ability of resistance to attacks. The contribution of W. Wang et al. "A Novel Encryption Algorithm Based on DWT and Multichaos Mapping" proposes a two-step discrete wavelet transform-based encryption algorithm enhanced with multichaos matrix. The authors proved that the proposed approach shows large key space, high key sensitivity, and ability of resistance to attacks.

In addition to encryption of data while in transit, privacypreserving data queries are crucial in wireless sensor network. The contribution of $\mathrm{H}$. Dai et al. "Random Secure Comparator Selection Based Privacy-Preserving MAX/MIN Query Processing in Two-Tiered Sensor Networks" proposed a privacy-preserving MAX/MIN query processing approach based on random secure comparator selection in two-tiered sensor network denoted as RSCS-PMQ.

Context awareness is a fundamental property of a system expressing the capacity of being aware of its physical environment or situation and responding in a proactive and intelligent manner. It is fundamental in the context of wireless sensor network for opening to advanced applications involving autonomous reasoning and decision-making. The contribution of M. Hussain et al. "CRAM: A Conditioned Reflex Action inspired Adaptive Model for Context Addition 
in Wireless Sensor Networks" proposed a context added system in which the actuations once performed by the system help the system itself to internally evolve enriching context repository through retrospective contexts and improving introspection.

We think that this special issue would shed light on major achievements in the area of embedded systems for mobile sensors and IoT and attract attention by the scientific community to pursue further investigations leading to the rapid development of these crucial technologies.

\section{Acknowledgments}

We would like to express our appreciation to all the authors for their informative contributions and the reviewers for their support and constructive critiques in making this special issue possible.

Marco Anisetti

Valerio Bellandi

Abdellah Chehri

Yurong Qian

Gwanggil Jeon 


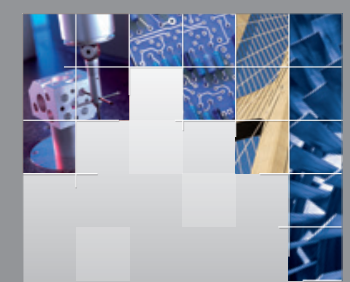

\section{Enfincering}
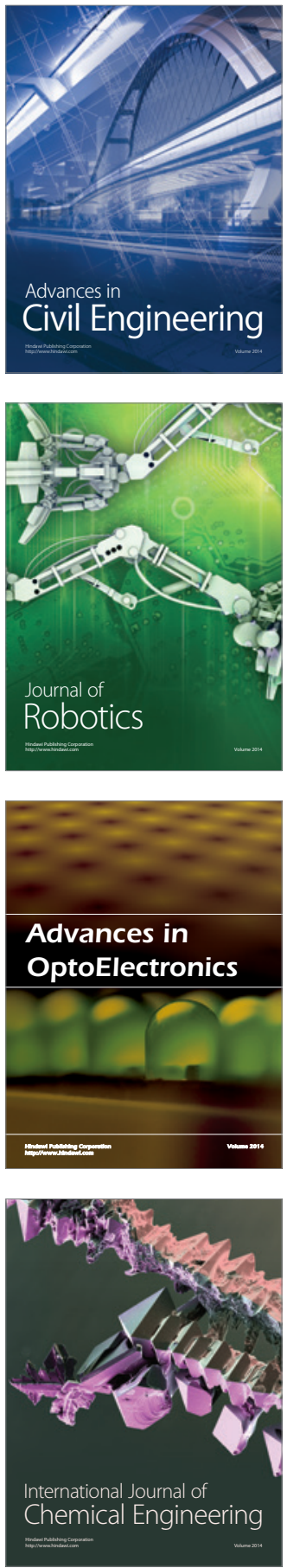

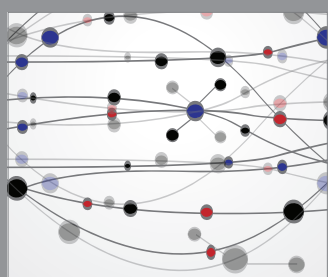

The Scientific World Journal

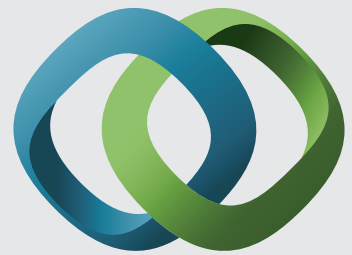

\section{Hindawi}

Submit your manuscripts at

http://www.hindawi.com
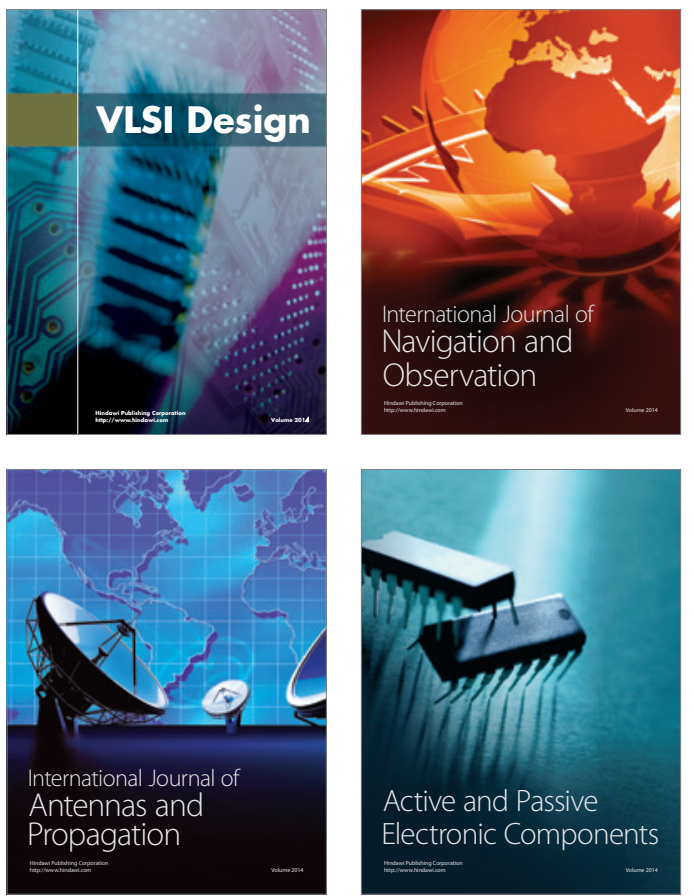
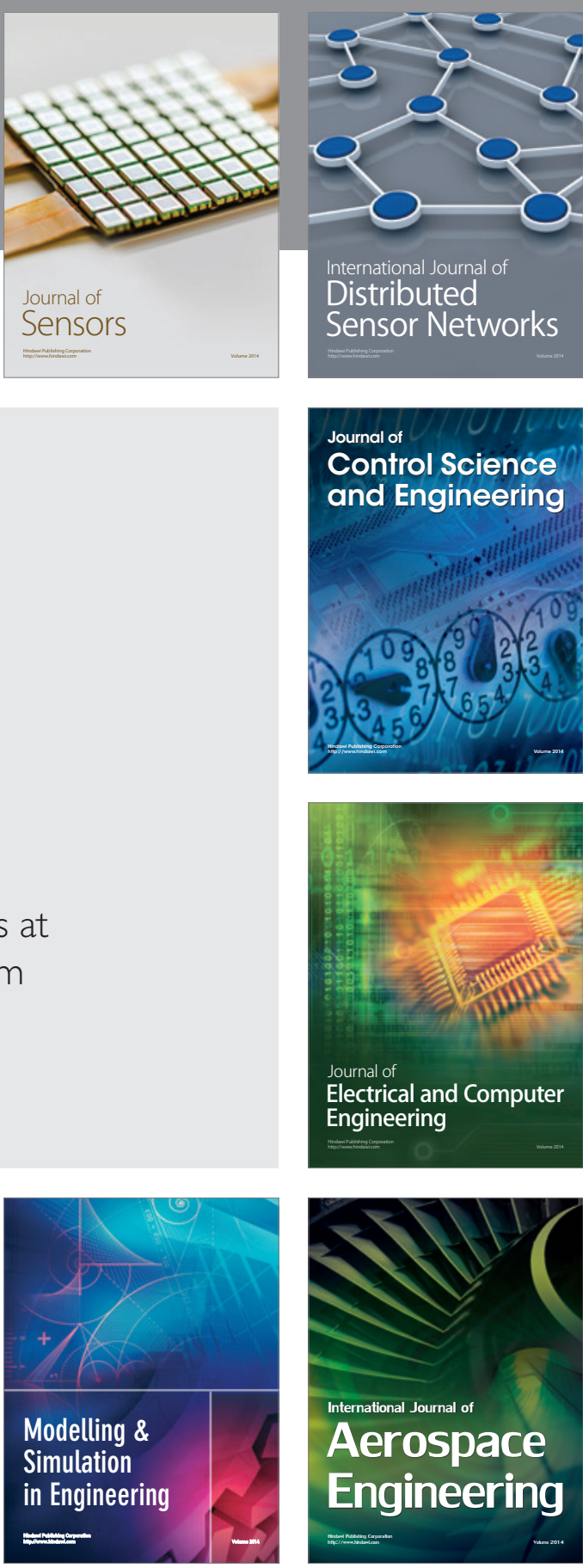

International Journal of

Distributed

Sensor Networks

Journal of

Control Science

and Engineering
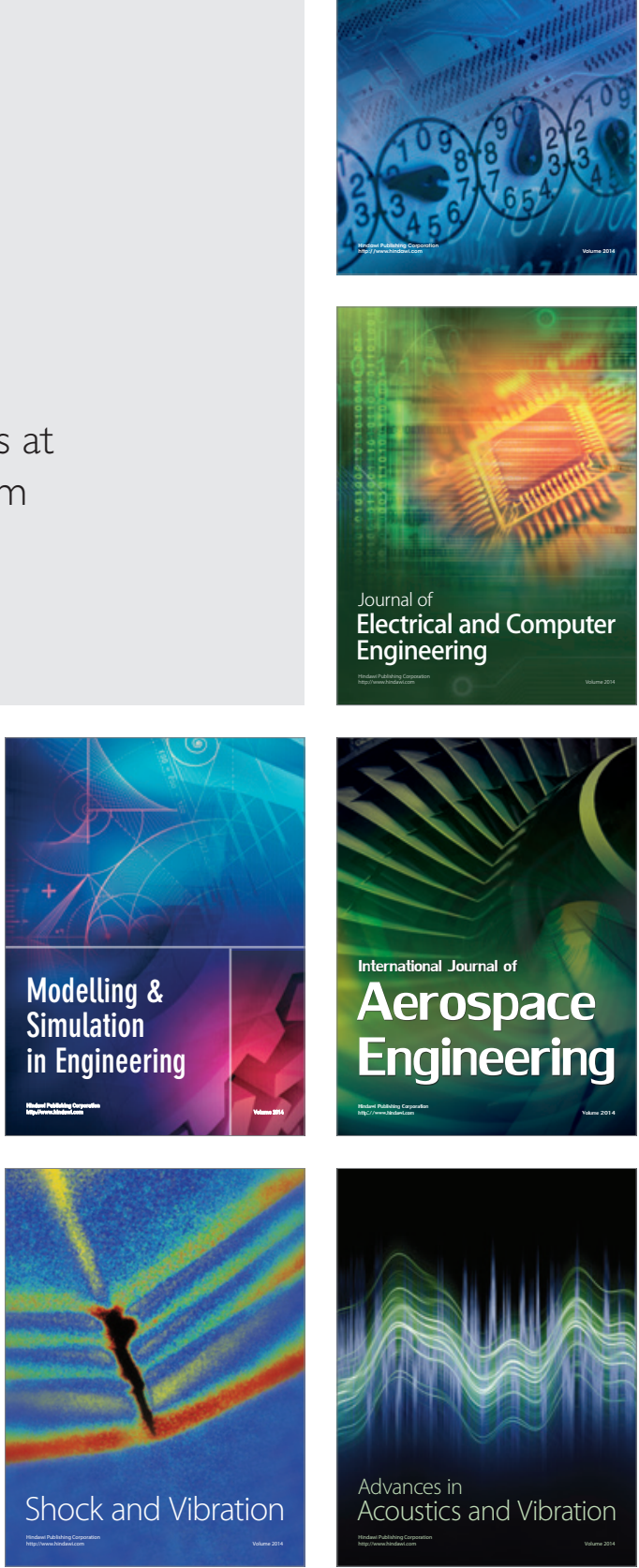